\title{
In search of temporal power laws in the orientational relaxation near isotropic-nematic phase transition in model nematogens
}

\author{
Prasanth P. Jose ${ }^{\text {a) }}$ and Biman Bagchib) \\ Solid State and Structural Chemistry Unit, Indian Institute of Science, Bangalore 560012, India
}

Recent Kerr relaxation experiments by Gottke et al. [J. Chem. Phys. 116, 360 (2002); 116, 6339 (2002)] have revealed the existence of a pronounced temporal power law decay in the orientational relaxation near the isotropic-nematic phase transition (INPT) of nematogens of rather small aspect ratio, $\kappa(\kappa \simeq 3-4)$. We have carried out very long (50 ns) molecular dynamics simulations of model (Gay-Berne) prolate ellipsoids with aspect ratio 3 in order to investigate the origin of this power law. The model chosen is known to undergo an isotropic to nematic phase transition for a range of density and temperature. The distance dependence of the calculated angular pair correlation function correctly shows the emergence of a long range correlation as the INPT is approached along the density axis. In the vicinity of INPT, the single particle second rank orientational time correlation function exhibits power law decay, $\left(t^{-\alpha}\right)$ with exponent $\alpha \sim 2 / 3$. More importantly, we find the sudden appearance of a pronounced power-law decay in the collective part of the second rank orientational time correlation function at short times when the density is very close to the transition density. The power law has an exponent close to unity, that is, the correlation function decays almost linearly with time. At long times, the decay is exponential-like, as predicted by Landau-de Gennes mean field theory. Since Kerr relaxation experiments measure the time derivative of the collective second rank orientational pair correlation function, the simulations recover the near independence of the signal on time observed in experiments. In order to capture the microscopic essence of the dynamics of pseudonematic domains inside the isotropic phase, we introduce and calculate a dynamic orientational pair correlation function (DOPCF) obtained from the coefficients in the expansion of the distinct part of orientational van Hove time correlation function in terms of spherical harmonics. The DOPCF exhibits power law relaxation when the pair separation length is below certain critical length. The orientational relaxation of a local director, defined in terms of the sum of unit vectors of all the ellipsoidal molecules, is also found to show slow power law relaxation over a long time scale. These results have been interpreted in terms of a newly developed mode coupling theory of orientational dynamics near the INPT. In the present case, the difference between the single particle and the collective orientational relaxation is huge which can be explained by the frequency dependence of the memory kernel, calculated from the mode coupling theory. The relationship of this power law with the one observed in a supercooled liquid near its glass transition temperature is explored.

\section{INTRODUCTION}

A system of elongated molecules is known to undergo a phase transition at low temperatures from an orientationally disordered, isotropic phase to an orientationally ordered, nematic phase, if the aspect ratio is larger than certain critical number. This isotropic to nematic phase transition (INPT) is a weakly first order phase transition and is characterized by a large growth of orientational pair correlation function near the transition point. However, the orientational pair correlation function does not diverge, because the impending divergence is preempted by the first order phase transition. ${ }^{1-13}$ These equilibrium characteristics make the study of orientational dynamics (both single particle and collective) near INPT highly interesting and theoretically challenging.

This paper addresses the origin of a recently observed,

\footnotetext{
a)Electronic mail: jose@sscu.iisc.ernet.in

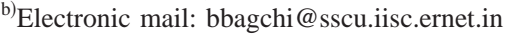

highly interesting power law decay of orientational correlation function near the isotropic-nematic transition (INPT) of several nematogens. Fayer and co-workers ${ }^{14-19}$ investigated the dynamics of nematogens from very short (few picosecond) to very long time scale (several hundred nanoseconds) as a function of temperature, using optical heterodyne detected Kerr effect. The data obtained from these experiments are related to impulse response function of the system which is the time derivative of the polarizability-polarizability time correlation function $C_{P}(t)$ [that is, the Kerr signal $\left.\propto-\left[d C_{P}(t) / d t\right]\right]$. The decay observed in this experiments is exponential on the longest time scale (beyond $100 \mathrm{~ns}$ ), which is well described by Landau-de Gennes theory. ${ }^{3,4}$ At short times, the decay is again exponential. However, at intermediate times (10 ps-10 ns), the data can be fitted to a temporal power law with an exponent, with a value less than unity. The value of the exponent is found to be independent of temperature, but to depend on the chemical identity of the 
nematogens (rather, on the aspect ratio), signaling (in the strict sense) a lack of universality of the type observed near a critical point. However, the values of exponent are all close to $2 / 3$ and thus some sort general behavior appears to be present in the dynamics. Gottke et al. also presented a mode coupling theory analysis which explained the emergence of the power law behavior as a consequence of the growing correlation length near I-N transition. ${ }^{16,17}$

Several computer simulation studies ${ }^{20-23}$ have earlier attempted to recover the power law in orientational relaxation near INPT. One of the first simulations to calculate second rank single particle and collective orientational time correlation function (OTCF) was performed by Allen et al. ${ }^{21}$ using hard ellipsoids. They could observe the critical slowing down of the dynamical correlation function near INPT, but did not observe any power law decay. Perera et $a .^{22}$ have done molecular dynamic simulation on a system of Gay Berne ellipsoids. This model undergoes an isotropic to nematic phase transition at a reduced density $\rho^{*} \simeq 0.315$ (where $\rho^{*}=\rho b^{3}, \rho$ being the number density and $b$ is the length of the minor axis of the ellipsoid) and temperature $T^{*} \simeq 1$ (where $T^{*}=k_{b} T / \epsilon_{0}$, while $k_{b}$ is the Boltzmann constant, $T$ is the temperature and $\epsilon_{0}$ is the energy constant used in the Gay-Berne potential) for aspect ratio $(\kappa=3)$. They found second rank single particle and collective OTCF slow down appreciably near INPT. In another set of simulations of Allen et al. ${ }^{23}$ calculated direct correlation functions of a system of Gay-Berne ellipsoids near INPT. Detailed molecular dynamic simulations of Ravichandran et al. ${ }^{22,24}$ found a sudden appearance of power law behavior in the second rank single particle OTCF near INPT. The value of power law exponent was close to 0.56 . These simulations also looked into the translational and rotational diffusion coefficient of a system of Gay-Berne ellipsoids. Vasanthi et al. ${ }^{25}$ carried out molecular dynamics simulations to calculate diffusion of isolated tagged spheres in a sea of Gay-Berne ellipsoids. The diffusion is isotropic well inside the isotropic region of nematic liquid crystal. In the vicinity of the INPT the parallel and perpendicular component of ( $D_{\|}$and $D_{\perp}$ ) diffusion coefficient decouples from each other. They proved that the anisotropy parameter $\left(D_{\|} / D_{\perp}\right)$ obeys a power law of the form $\left(\rho_{c}^{*}-\rho^{*}\right)^{p}$.

However, all the simulations reported until now have failed to detect the temporal power law in the decay of collective orientational correlation function, which, as already mentioned, is the experimental quantity one measures in Kerr relaxation experiments (actually one measures the time derivative of the second rank orientational time correlation function). Perhaps, the reason is that these early simulations were limited in the duration of the trajectory obtained. Or, may be, the intermolecular potential employed was inadequate. The nonobservance of the power law decay in simulations has remained somewhat of a puzzle.

Theoretically, two limits of orientational relaxation near INPT are understood clearly. The long time decay is expected to be given by the Landau-de Gennes theory which predicts an exponential decay of the second rank orientational correlation function

$$
C_{20}^{\mathrm{LdG}}=\exp \left(-t / \tau_{\mathrm{LdG}}\right) .
$$

The Landau-de Gennes time constant $\tau_{\mathrm{LdG}}$ shows a pronounced increase near the I-N transition. The physical origin of this slow down in relaxation is easily understood from a mean-field theory which gives the following expression for $\tau_{\mathrm{LdG}}:$

$$
\tau_{\mathrm{LdG}}=\frac{S_{220}(k=0)}{6 D_{R}},
$$

where $S_{220}(k)$ is the wave number dependent orientational structure factor in the intermolecular frame (with $k$ parallel to the $Z$ axis) and where the subscript 220 denotes the orientational correlation of spherical harmonics $\left(Y_{\ell, m}\right)$ of rank $\ell=2$ and azimuthal number $m=0 . D_{R}$ is the long time rotational diffusion coefficient of the nematogens in the isotropic phase. As the I-N transition is approached, $S_{220}(k=0)$ becomes very large, reflecting the appearance of long range orientational correlation near the phase transition. Note that $S_{220}(k=0)$ does not actually diverge because of the intervention of the (weakly) first order I-N phase transition.

While the Landau-de Gennes mean field theory is expected to be valid in the long time (at times much larger than $6 D_{R}^{-1}$ ), the early short time decay of the orientational time correlation function should be dominated by the short range local forces. Here the decay rate should be close to $6 D_{R}^{0}$, where $D_{R}^{0}$ is the short time limit of the rotational diffusion coefficient. This rate is, for the systems studied by Fayer and co-workers, is of the order of $10^{11} \mathrm{~s}^{-1}$ or so. Note that the short time rotational diffusion coefficient $D_{R}^{0}$ is expected to be much larger than the long time diffusion coefficient, because the former is determined by the local interactions while the latter contains the effects of slow density fluctuations. The long time Landau-de Gennes exponential decay is expected to set at times (much) longer than ns. It is the intermediate time range between, say, $10 \mathrm{ps}$ and $10 \mathrm{~ns}$, which is of interest in this study. In this time range, the simple mean-filed theory is not expected to be valid because the memory function or the rotational friction should be a strong function of frequency. Mode coupling theory predicts interesting dynamical behavior at such intermediate times. It suggests that due to rapid increase in the static two particle orientational correlation as the $\mathrm{I}-\mathrm{N}$ transition is approached, the friction on individual rotors also increases rapidly. This effect is felt at intermediate times when the singular part of the friction is found to behave as ${ }^{16,17}$

$$
\Gamma^{\operatorname{sing}}(t) \approx A_{0} / \sqrt{t} .
$$

Orientational time correlation function is given as ${ }^{16,17}$

$$
C_{20}(t) \approx \exp \left(a^{2} t\right) \operatorname{erfc}(a \sqrt{t})
$$

where erfc is a complementary error function and the quantity a is determined by a combination of orientational structure factor and rotational diffusion coefficient. As mentioned earlier, the above expression is predicted to be valid in the time window which is short compared to $\tau_{\mathrm{LdG}}$ but long compared to $\left(6 D_{R}\right)^{-1}$. Complementary error function has the following asymptotic expansion: 


$$
\operatorname{erfc}(x) \approx \frac{e^{-x^{2}}}{\sqrt{\pi} x}\left(1-\frac{1}{2 x^{2}}+\frac{1.3}{\left(2 x^{2}\right)^{2}}+\frac{1.3 .5}{\left(2 x^{2}\right)^{3}}+\cdots\right)
$$

Thus, the leading term in the expansion varies as $t^{-1 / 2}$. As shown by Gottke et al., MCT prediction [Eq. (4)] seems capable of describing the experimentally observed decay over two orders of magnitude but not over the entire range of the observed slow decay. ${ }^{16,17}$

Therefore, not only does the MCT predict a weak time dependence at intermediate times, it also gives a clear physical picture of the origin of this slow decay. At short times, of the order of $1-10$ ps or less, orientational relaxation is determined by local dynamics. But at somewhat longer times (that is, beyond $10 \mathrm{ps}$ or so), orientational relaxation requires relaxation of the nearest neighbors. In quantitative terms, this implies that the friction on rotational motion is coupled to the isotropic and orientational density fluctuations of domains. However, fluctuations of these domains are slow to relax, especially near the INPT. As a consequence, the friction due to the presence of the nonlocal, pseudonematic density fluctuations increases with time, that is with lowering frequency. The increase in friction at intermediate times is sufficiently fast to slow down the relaxation to a great extent. After certain time, when the increase of friction slows down, the relaxation occurs with constant friction and we get back the exponential decay. This last stage is the Landau-de Gennes regime. Thus, MCT predicts two crossover regionsone from a fast, short time exponential decay to a power-law regime of slow decay and a second one from power law to, even slower but exponential decay. The duration of the weak time dependence in the intermediate time is predicted to depend on the duration of the growth of the friction. This duration of the growth of the friction is correlated with the length of the long range correlation present in the system. Thus, we expect the duration of the persistence of the power law to increase as the INPT is approached. Note that $\tau_{\mathrm{LdG}}$ also increases simultaneously because $S_{220}(k \approx 0)$ becomes very large. In addition, since the Kerr relaxation experiments measure the time derivative of the polarizabilitypolarizability time correlation function, a very weak time dependence in experiments implies a nearly linear time dependence of the second rank, collective, orientational correlation function.

In this work we carried out very long time (approximately $50 \mathrm{~ns}$ in real time unit) molecular dynamics simulations of larger systems (than attempted earlier) to calculate the second rank collective OTCF of a system of Gay-Berne ellipsoids. We find that just prior to INPT, the relaxation of second rank collective OTCF $\left[C_{2}^{c}(t)\right]$ [defined in Eq. (10)], abruptly changes its decay behavior from exponential to a pronounced power law decay which ranges from short to intermediate times. The decay again changes over to an exponential-like decay at longer times.

In order to gain a microscopic understanding of the power law decay near the INPT, we have looked into the study of two other time correlation functions. First, we investigated the relaxation of the dynamic orientational pair correlation function (DOPCF) and, second we studied the OTCF of a local director. The DOPCF can probe orienta- tional dynamics of a pair of ellipsoids at different length scales; this gives a more detailed description of the relaxation of the orientational order than the collective OTCF. The DOPCF shows critical slowing down of relaxation and the emergence of power law relaxation near INPT. The emergence of power law relaxation is also evident in the OTCF of the local director that is discussed in the appendix. Present simulations also explore the divergence of various equilibrium angular pair correlation functions which measure the growth of correlation length near INPT.

The next section of this paper defines various static and dynamical correlation functions. Section III gives the details of the molecular dynamics simulations. Results obtained from the simulations are discussed in Sec. IV. Concluding remarks are given in Sec. V.

\section{STATIC AND DYNAMIC ORIENTATIONAL CORRELATION FUNCTIONS}

The radial distribution function for nonspherical molecules in a laboratory fixed frame can be expressed in terms of average over delta functions as ${ }^{26,27}$

$$
\begin{aligned}
g\left(\mathbf{r}, \boldsymbol{\Omega}, \boldsymbol{\Omega}^{\prime}\right)= & \frac{1}{N \rho}\left\langle\sum_{i}^{N} \sum_{j}^{N^{\prime}} \delta\left(\mathbf{r}+\mathbf{r}_{j}-\mathbf{r}_{i}\right) \delta\left(\boldsymbol{\Omega}-\boldsymbol{\Omega}_{i}\right)\right. \\
& \left.\times \delta\left(\boldsymbol{\Omega}^{\prime}-\boldsymbol{\Omega}_{j}\right)\right\rangle
\end{aligned}
$$

(the prime over the summation signifies that terms $i=j$ are excluded from the sum), where $\mathbf{r}_{i}$ is the translational coordinate in the arbitrary reference system and $\boldsymbol{\Omega}_{i}$ is the polar angle for the $i$ th linear molecule in the laboratory fixed reference frame. This pair correlation function can be conveniently represented in an intermolecular reference frame where $z$ axis passes through the center of mass of two ellipsoids. The pair correlation function in this reference frame is given as $g\left(r, \omega, \omega^{\prime}\right)$. It gives the joint probability to find any two ellipsoids separated by a distance $r$ and with angular coordinates $\omega$ and $\omega^{\prime}$. This orientational correlation function can be expanded in spherical harmonics ${ }^{26,27}$

$$
g\left(r, \omega, \omega^{\prime}\right)=\sum_{l_{1}, l_{2}, m} g_{l_{1} l_{2} m}(r) Y_{l_{1}, m}(\omega) Y_{l_{2}, \underline{m}}\left(\omega^{\prime}\right),
$$

i.e., the $\omega=(\theta, \phi)$ and $\omega^{\prime}=\left(\theta^{\prime}, \phi^{\prime}\right)$ are the angular coordinates in the intermolecular frame. $\theta$ defines the angle an ellipsoid makes with the $z$ axis of the intermolecular frame and $\phi$ defines the angle an ellipsoid makes with the $x$ axis in the $x-y$ plane of the intermolecular frame. The angular pair correlation functions are obtained from the total pair correlation function using the orthogonal properties of the spherical harmonics. ${ }^{26}$ These angular pair correlation functions can be calculated from simulation using the expression ${ }^{26}$

$$
g_{l_{1} l_{2} m}(r)=16 \pi^{2}\left\langle g\left(r, \omega, \omega^{\prime}\right) Y_{l_{1}, m}^{*}(\omega) Y_{l_{2}, \underline{m}}^{*}\left(\omega^{\prime}\right)\right\rangle \text {. }
$$

The distance dependence of angular pair correlation function gives the range of orientational order in among the molecules in a system of nematogens.

Time dependent orientational correlation function gives a measure of the temporal decay of the memory of orienta- 
tional order and one needs to define several such functions to describe various aspects of complex orientational dynamics. The single particle OTCF gives a temporal measure of loss of a single molecule's memory of its orientation in the random potential created by the surrounding molecules in the liquid environment. The single particle OTCF of the rank $l$ is defined as ${ }^{21,22,24,28,29}$

$$
C_{l}^{s}(t)=\frac{\left\langle\Sigma_{i} P_{l}\left(\hat{e}_{i}(0) \cdot \hat{e}_{i}(t)\right)\right\rangle}{\left\langle\sum_{i} P_{l}\left(\hat{e}_{i}(0) \cdot \hat{e}_{i}(0)\right)\right\rangle},
$$

where the $\hat{e}_{i}$ is the unit vector or director associated with $i$ th ellipsoid along the major axis of the ellipsoid and $P_{l}$ is $l$ th rank Legendre polynomial. The quantity measured in light scattering experiments is the collective OTCF. ${ }^{30}$ The collective OTCF is defined as ${ }^{21,22,27-29}$

$$
C_{l}^{c}(t)=\frac{\left\langle\Sigma_{i} \Sigma_{j} P_{l}\left(\hat{e}_{i}(0) \cdot \hat{e}_{j}(t)\right)\right\rangle}{\left\langle\Sigma_{i} \Sigma_{j} P_{l}\left(\hat{e}_{i}(0) \cdot \hat{e}_{j}(0)\right)\right\rangle} .
$$

This is a limiting case of the more general wave number $(k)$ dependent orientational correlation function $C_{l}^{c}(k, t)$ defined as

$$
C_{l}^{c}(k, t)=\frac{\left\langle\sum_{i} \sum_{j} e^{i \mathbf{k} \cdot \mathbf{r}_{i j}} P_{l}\left(\hat{e}_{i}(0) \cdot \hat{e}_{j}(t)\right)\right\rangle}{\left\langle\sum_{i} \sum_{j} e^{i \mathbf{k} \cdot \mathbf{r}_{i j}} P_{l}\left(\hat{e}_{i}(0) \cdot \hat{e}_{j}(0)\right)\right\rangle} .
$$

A wealth of information about the space dependence of orientational relaxation can be obtained by studying the two particle correlation functions, such as molecular van $\mathrm{Hove}^{27}$ correlation function. In order to study the orientational dynamics of a pair of particles we have defined a DOPCF. Orientational pair correlation function is obtained from the distinct part of Van Hove correlation function by expanding it in terms of spherical harmonics. Van Hove correlation function for a molecular liquid can be defined as

$$
\begin{aligned}
G_{d}\left(\mathbf{r}, \boldsymbol{\Omega}, \boldsymbol{\Omega}^{\prime}, t\right)= & \frac{1}{N}\left\langle\sum_{i}^{N} \sum_{j}^{N^{\prime}} \delta\left(\mathbf{r}+\mathbf{r}_{j}(0)-\mathbf{r}_{i}(t)\right)\right. \\
& \left.\times \delta\left(\boldsymbol{\Omega}-\boldsymbol{\Omega}_{i}(0)\right) \delta\left(\boldsymbol{\Omega}^{\prime}-\boldsymbol{\Omega}_{j}(t)\right)\right\rangle .
\end{aligned}
$$

In a system of linear molecules, the van Hove correlation function can be represented in intermolecular reference frame as $G_{d}\left(r, \omega, \omega^{\prime}, t\right)$. This correlation function can be expanded in terms of spherical harmonics to get DOPCF,

$$
G_{d}\left(r, \omega, \omega^{\prime}, t\right)=\sum_{l_{1}, l_{2}, m} \rho G_{l_{1} l_{2} m}(r, t) Y_{l_{1}, m}(\omega) Y_{l_{2}, \underline{m}}\left(\omega^{\prime}\right) .
$$

The DOPCF gives a direct measure of relaxation of orientational order at different shells around a linear molecule. The DOPCF can be calculated from the simulation using the expression

$$
G_{l_{1} l_{2} m}(r, t)=16 \pi^{2}\left\langle G_{d}\left(r, \omega, \omega^{\prime}, t\right) Y_{l_{1}, m}^{*}(\omega) Y_{l_{2}, \underline{m}}^{*}\left(\omega^{\prime}\right)\right\rangle / \rho .
$$

In order to study the orientational relaxation of different cages, we can define orientational correlation function in terms of DOPCF. Let

$$
C_{p}(t)=\left.\frac{G_{l_{1} l_{2} m}(r, t)-G_{l_{1} l_{2} m}(r, t=\infty)}{G_{l_{1} l_{2} m}(r, t=0)-G_{l_{1} l_{2} m}(r, t=\infty)}\right|_{r=R},
$$

where $R$ is a fixed distance at which the relaxation is measured.

\section{DETAILS OF MOLECULAR DYNAMICS SIMULATIONS}

Long molecular dynamics simulations have been carried out for a system of 576 Gay-Berne ellipsoids ${ }^{31}$ in a microCanonical ensemble. The form of the modified intermolecular Gay-Berne potential is $22,24,25,32$

$$
\begin{aligned}
U= & 4 \epsilon\left(\hat{r}, \vec{u}_{i}, \vec{u}_{j}\right)\left[\left(\frac{\sigma_{s}}{r-\sigma\left(\hat{r}, \vec{u}_{i}, \vec{u}_{j}\right)+\sigma_{s}}\right)^{12}\right. \\
& \left.-\left(\frac{\sigma_{s}}{r-\sigma\left(\hat{r}, \vec{u}_{i}, \vec{u}_{j}\right)+\sigma_{s}}\right)^{6}\right],
\end{aligned}
$$

where $\hat{r}$ is the unit vector that passes through the center of mass of a pair of molecules, $\vec{u}_{i}$ and $\vec{u}_{j}$ unit vectors that passes through the major axis of a pair of ellipsoidal molecules. $\epsilon$ and $\sigma$ give the strength and range of interaction,

$$
\begin{aligned}
\sigma\left(\hat{r}, \vec{u}_{i}, \vec{u}_{j}\right)= & \sigma_{s}\left[1-\frac{\chi}{2}\left(\frac{\left(\vec{u}_{i} \cdot \hat{r}+\vec{u}_{j} \cdot \hat{r}\right)^{2}}{1+\chi\left(\vec{u}_{i} \cdot \vec{u}_{j}\right)}\right.\right. \\
& \left.\left.+\frac{\left(\vec{u}_{i} \cdot \hat{r}-\vec{u}_{j} \cdot \hat{r}\right)^{2}}{1-\chi\left(\vec{u}_{i} \cdot u_{j}\right)}\right)\right],
\end{aligned}
$$

$\sigma_{s}$ is double of the minor axis $b, \kappa$ gives molecular elongation (aspect ratio), which is the ratio of end-to-end to sideto-side diameters, $\kappa=\sigma_{e} / \sigma_{s}$. we have worked with aspect ratio is 3 . In the expression for $\sigma, \chi$ can be given in terms of $\kappa$ as $\chi=\left(\kappa^{2}-1\right) /\left(\kappa^{2}+1\right)$,

$$
\begin{aligned}
\epsilon\left(\hat{r}, \vec{u}_{i}, \vec{u}_{j}\right)= & \epsilon_{0}\left[1-\chi^{2}\left(\vec{u}_{i} \cdot \vec{u}_{j}\right)\right]^{-1 / 2}[1 \\
& \left.-\frac{\chi^{\prime}}{2}\left(\frac{\left(\vec{u}_{i} \cdot \hat{r}+\vec{u}_{j} \cdot \hat{r}\right)^{2}}{1+\chi\left(\vec{u}_{i} \cdot \vec{u}_{j}\right)}+\frac{\left(\vec{u}_{i} \cdot \hat{r}-\vec{u}_{j} \cdot \hat{r}\right)^{2}}{1-\chi\left(\vec{u}_{i} \cdot u_{j}\right)}\right)\right]^{2},
\end{aligned}
$$

where $\epsilon_{0}$ is the energy parameter and $\chi^{\prime}=\left(\sqrt{\kappa^{\prime}}-1\right) /\left(\sqrt{\kappa^{\prime}}\right.$ $+1)\left(\kappa^{\prime}=\epsilon_{s} / \epsilon_{e}\right.$ gives the strength of interaction which is side-to-side to end-to-end well depths). The value of $\kappa^{\prime}$ used in the simulation is $5 .{ }^{24}$ The scaling used for moment of inertia is $I^{*}=I / m \sigma_{0}^{2}$. The density is scaled in the simulation as $\rho^{*}=\rho \sigma_{0}^{2}$ and the temperature is scaled as $T^{*}=k_{b} T / \epsilon_{0}$. The equation of motion is integrated with reduced time $\left[t^{*}\right.$ $\left.=\left(m \sigma_{0}^{2} / \epsilon_{0}\right)^{1 / 2}\right]$ steps with $\Delta t=0.002 t^{*}$.

The simulation starts from an equilibrated configuration of ellipsoids. Initial configuration of the ellipsoids is generated from a cubic lattice and then the simulation is run for 200000 steps to obtain the equilibrium configuration. During the equilibration steps the temperature is scaled so that the system is in equilibrium at this particular temperature. The production steps starts from this equilibrated configuration. The production steps are run for 15 million steps to calculate statistically averaged single particle and collective static ${ }^{33-35}$ and dynamic orientational correlation functions. When trans- 


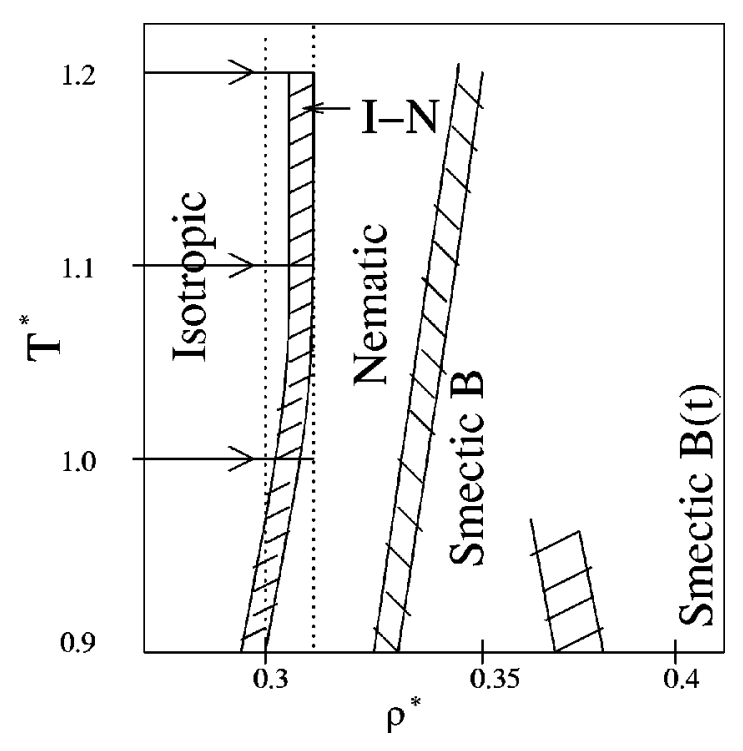

FIG. 1. A region of phase diagram of the Gay-Berne ellipsoids taken from Fig. 10 of Ref. 32 near INPT where simulations presented here are done. Shaded portion are the phase coexistence regions. The region between the dotted lines are the region of the phase diagram between density $\rho^{*}=0.3$ and $\rho^{*}=0.315$ where the pretransition effects were found. Arrows denote temperatures at which density has been varied toward the transition.

lated into argon units, this corresponds to a run time of $75 \mathrm{~ns}$. We found that such long runs are indeed necessary to study decay of collective orientational correlation near the INPT.

The ellipsoid used in the simulation has minor axis $b$ $=0.5$ and major axis $a=1.5$ (in reduced units). The order parameter of the system can be written in terms of three orthogonal vectors $\mathbf{a}, \mathbf{b}, \mathbf{c}$ associated with the molecule. Then the tensorial order parameter of INPT is defined as ${ }^{4,6}$

$$
S_{i j}^{\alpha \beta}=\frac{1}{2}\left\langle 3 i_{\alpha} j_{\beta}-\delta_{\alpha \beta} \delta_{i j}\right\rangle,
$$

where $\alpha, \beta=x, y, z$ are indices referring to the laboratory frame, while $i, j=a, b, c$, and $\delta_{\alpha \beta}$ and $\delta_{i j}$ are Kronecker delta symbols. Here $S_{i j}^{\alpha \beta}$ is diagonalized and it is the highest absolute value that is taken as the order parameter $(S)$.

The simulations are done at the state points near the pretransition region of phase diagram, shown in Fig. 1 where the variation in temperature and the density employed is shown by arrows. The translational and rotational motions are solved using leap-frog algorithm. The calculated order parameter is shown in Fig. 2 where the order parameter is plotted against the density near INPT at three temperatures. The order parameter changes dramatically after density increases beyond 0.3. This is in accord with previous simulations. $^{22-25}$

\section{RESULTS AND DISCUSSIONS}

\section{A. Static pair correlation functions}

As already mentioned, the INPT occurs near $\rho^{*} \geqslant 0.3$ for all temperatures used in this simulation. Figures 3(a), 3(b), and 3(c) show the distance dependence of angular pair correlation functions plotted against position for a system of Gay-Berne ellipsoids at temperature 1.0 for different number densities, $\rho^{*}=0.285-0.315$. The angular correlation function $g_{200}(r)$ [plotted in Fig. 3(a)] shows the sharpening

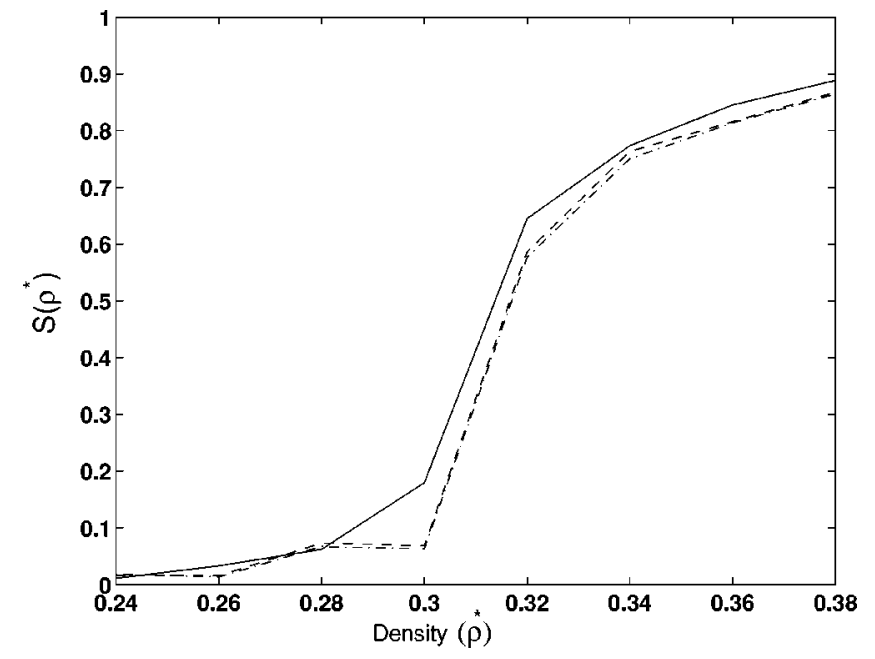

FIG. 2. The variation of order parameter $\left[S\left(\rho^{*}\right)\right]$ with density $\rho^{*}$ at different temperatures is shown in this figure. The continuous line gives the $S\left(\rho^{*}\right)$ at $T^{*}=1.0$, the dashed line gives the $S\left(\rho^{*}\right)$ at $T^{*}=1.1$ and the dashed-dotted line gives $S\left(\rho^{*}\right) T^{*}=1.2$.

of the peaks as the INPT is approached. This angular pair correlation function correlates angular distribution of ellipsoids around a test sphere. Since the orientation of only one of the ellipsoid is taken into consideration in the calculation of $g_{200}(r)$, this angular pair correlation function does not diverge even in the nematic phase. But the sharpening of peaks of this correlation function shows the building up of orientational order when the nematic phase is approached along the density axis. The angular pair correlation function $g_{220}(r)$ which measures the correlation between a pair of ellipsoids separated by a distance $r$, shows divergence in the

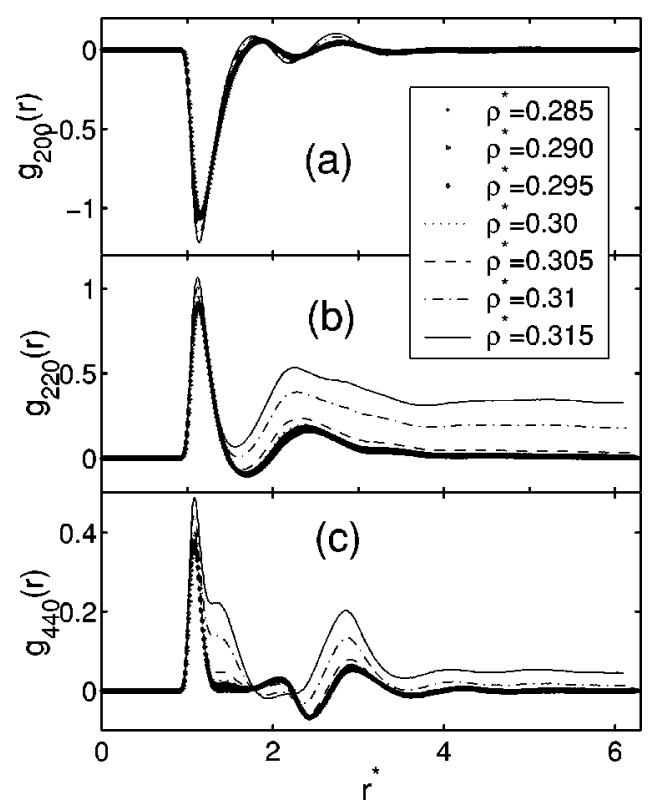

FIG. 3. Three angular pair correlation functions are plotted at different densities against distance of separation at $T^{*}=1.0$. (a) shows $g_{200}(r)$ angular pair correlation function. (b) gives the $g_{220}(r)$ angular pair correlation function. This is the first angular pair correlation function which shows significant divergence near INPT. The angular pair correlation function $g_{440}(r)$ is shown in (c). 


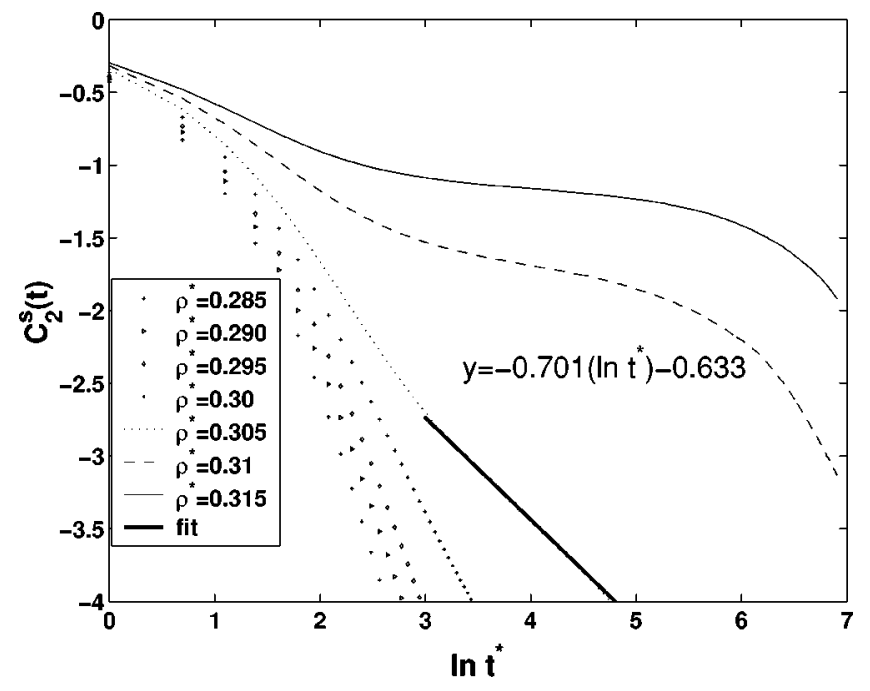

FIG. 4. The $\log -\log$ plot of single particle OTCF $\left[C_{2}^{s}(t)\right]$ [Eq. (9)], at different densities is shown here. The linear region of the relaxation function signifies the emergence of a power law relaxation after $\rho^{*}=0.3$. The linear portion of the $C_{2}^{s}(t)$ is fitted to a straight line at density $\rho^{*}=0.305$.

vicinity of INPT as depicted in Fig. 3(b). The divergence in angular pair correlation function is also evident in higher order correlation functions, as shown in the Fig. 3(c). Note that these higher order correlations have non-negligible contribution in the series expansions of total angular correlation function $g\left(r, \omega, \omega^{\prime}\right)$ [Eq. (7)]. Therefore, the truncation of the series expansion of angular pair correlation can create non-negligible error in the total angular pair correlation function. Also note that for the system sizes simulated here, the correlation length of angular correlation is comparable to the size of the system. As the system approaches INPT whole system starts to behave like a single pseudonematic domain.

\section{B. Dynamics}

We now turn to dynamics of orientational relaxation. The $\log -\log$ plot of second rank single particle OTCF against reduced time at different densities $\rho^{*}=0.285$ to 0.315 is shown in Fig. 4. The power law relaxation emerges in the correlation function when the reduced density increases beyond $\rho^{*}=0.3$. In this region, the decay of the correlation function in the intermediate times can be represented by the function of the form $X(t) \simeq b t^{-a}$, where $a$ and $b$ are constants. At higher densities the fast and slow relaxation processes are clearly separated by intermediate power law as indicated by the linear region of the curve (between 20 and 100 in reduced unit). In Fig. 4 the linear region of the $\log -\log$ plot is fitted to a straight line,

$$
\ln (X(t))=b-a \ln (t),
$$

the exponent obtained from the fit is $a=0.70$. As the density increases the single particle orientational relaxation becomes slower due to the strong coupling of rotational motion with the surrounding ellipsoids or due to an orientational caging effect of the ellipsoids. The formation of orientational cage or pseudonematic region surrounding an ellipsoid gives it the memory of the previous orientation resulting in the slow relaxation process. The collective relaxation of the ellipsoids

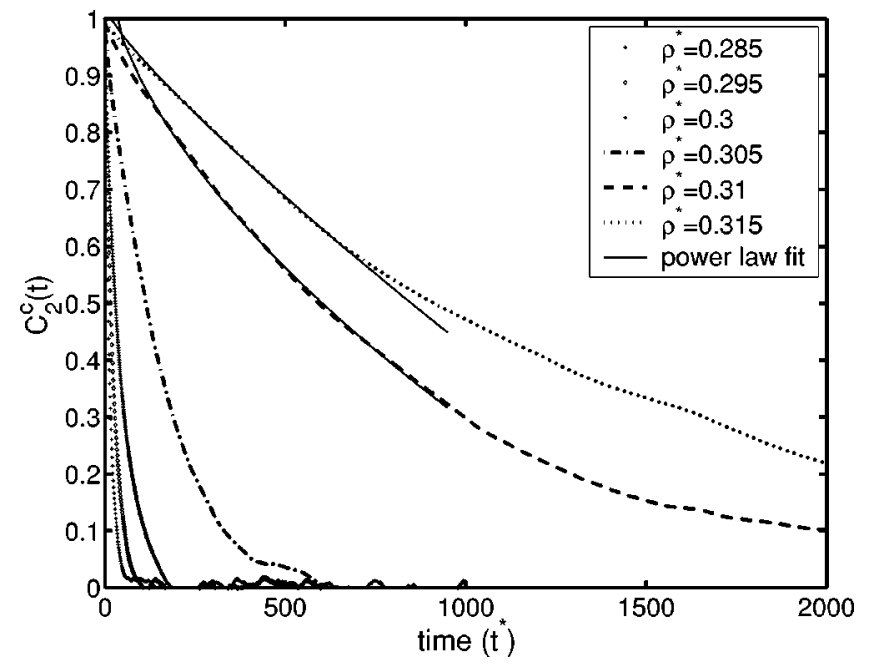

FIG. 5. The second rank collective OTCF, $\left[C_{2}^{c}(t)\right.$, Eq. (10)] is plotted against time at different densities and at constant temperature $T^{*}=1$. As the density approaches INPT this correlation function shows power law relaxation. The regions where power law relaxation is dominant are fitted with the function $y=1.1-0.014 t^{0.58}$ at $\rho^{*}=0.31$ and $y=1.0-0.0016 t^{0.85}$ at $\rho^{*}$ $=0.315$.

that form an pseudonematic domain is important near this state point (near 0.3 of the phase diagram shown in the Fig. 1).

The collective OTCF function at densities $\rho^{*}$ ranging from 0.285 to 0.315 are plotted in Fig. 5. The collective OTCF also shows significant lengthening in the time scale of relaxation. The change in the time scale of the relaxation is of the order of hundreds when density changes from 0.285 to 0.315 . At very close to the INPT, the nature of the decay of collective orientational relaxation function changes abruptly. We note that this observation of slow down of the of relaxation of the collective orientational relaxation is in general agreement with Landau-de Gennes mean field theory at longer time scale. The emergence of the power law is evident in this correlation function at densities $\rho^{*}=0.31$ (the value of the exponent obtained is 0.58 ) and $\rho^{*}=0.315$ (the value of the exponent obtained is 0.85 ) from the power law fit at the initial part of these correlation functions.

In Fig. 6 we have plotted the logarithm of the time derivative of the second rank collective orientational correlation function (the experimentally measured quantity) against logarithm of time to further our comparison with the observed behavior. The derivative has been plotted for two densities in the transition region. This figure brings out the crossover from the power law to the exponential decay at long times rather nicely. Also, this figure can be compared with Fig. 5 of Cang et al. ${ }^{19}$ Over more than two orders of magnitude, the similarity is rather remarkable.

We have earlier discussed the explanation put forward by using the mode coupling theory of relaxation near the INPT. According to this interpretation, the power law decay arises because of the rapid increase in the rotational memory function as the frequency decreases. In other words, subsequent to the initial relaxation within the cage, any collective (or single particle) relaxation becomes coupled to the orientational density fluctuation which becomes very slow near 


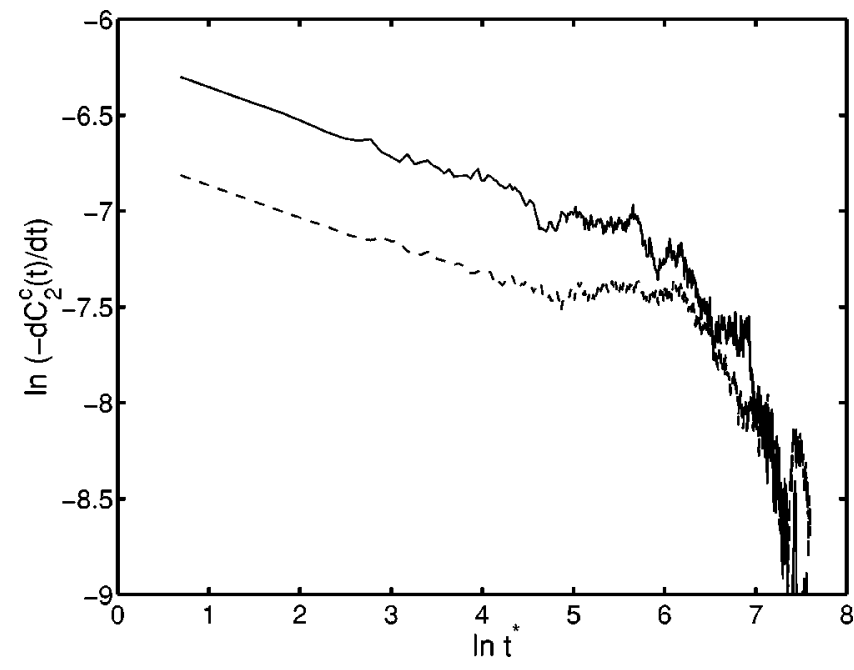

FIG. 6. The log of negative of the time derivative of second rank collective OTCF, $\left[C_{2}^{c}(t)\right.$ of Eq. (10)], is plotted for densities $\rho^{*}=0.31$ (dashed line) and $\rho^{*}=0.315$ (continuous line) against $\log$ of time at $T^{*}=1.0$. The linear portion of the plot gives power law observed in the intermediate time scale of the experiment.

the INPT because of the emergence of the long range orientational pair correlation. Thus, the reason for the power law decay in the collective and the single particle decay is nearly the same. However, the time scales of the two decay are vastly different - the former is much slower. The reason can be understood from the mean-field theory itself. Unlike the single particle dynamics, the collective orientation occurs in a collective many-body potential. For small density fluctuation, this potential can be approximated as a harmonic one. As the INPT is approached, the frequency of the harmonic potential decreases rapidly, slowing down relaxation in the well.

In many aspects orientational relaxation near INPT looks similar to that in the supercooled liquid near the glass transition. ${ }^{16,17}$ As discussed earlier, the reasons are also similar. Both is a reflection of a rapid increase in the memory function at low frequency. However, the reason for this rapid increase is different in the two cases. Now it is interesting to look into the orientational relaxation within various shells. This gives a close picture of the dynamics at varying length scales. Such information can be obtained from the dynamic pair correlation function. Figure 7 shows the behavior of DOPCF $G_{220}(r, t)$ at three densities at three different time scales. This gives a picture of slowing down of orientational relaxation near INPT. Note that in the top figure DOPCF at time $t=0$ converge to zero at large distance, signifying the absence of long-range orientational order. The picture of relaxation changes at higher densities. At $\rho^{*}=0.315$, the DOPCF is nonzero at time $t=0$ even at long distance. At this density the slow orientation relaxation can be identified from the widely separated dashed-dotted (time scale of tens) lines and dashed lines (time scale separated by hundreds). The orientation relaxation at the first peak of DOPCF is denoted by $C_{p}(t)$ [Eq. (15)]. In Fig. 8 the $C_{p}(t)$ is plotted at $R$ equal to first peak of DOPCF. When density is at 0.305 the sudden appearance of the power law is observed. In Fig. 8 the linear portion of the $C_{p}(t)$ is fitted to a straight line. The exponent

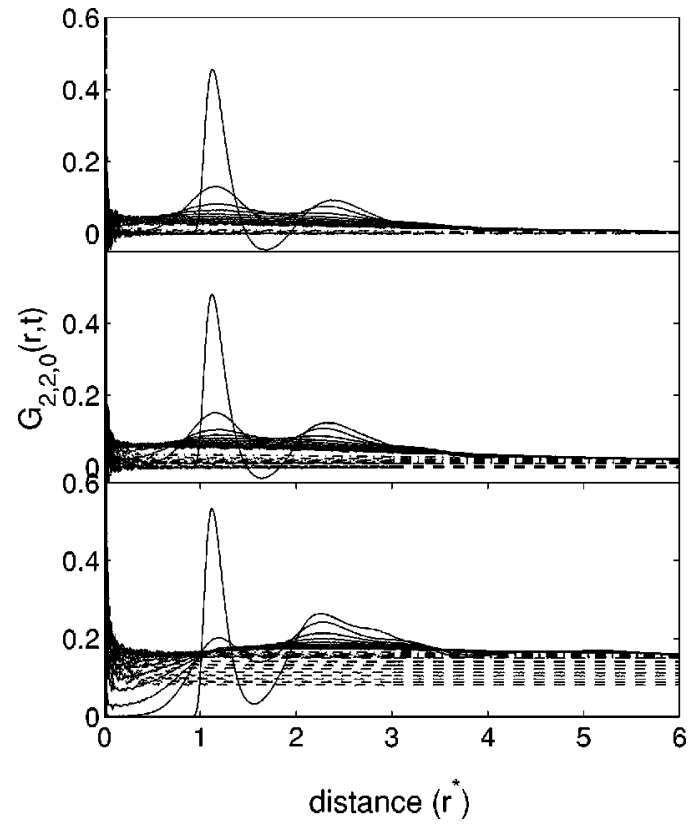

FIG. 7. The dynamic orientational pair correlation function (DOPCF) [Eq. (14)] is plotted at three different densities. In these figure continuous lines starting from the top are plotted at time step of 1 in the time interval 1-10. Then dotted-dashed lines are plotted at a time step of 10 in the time interval 20-100. Finally the dashed lines are plotted at a time step of 100 in time interval 200-1000. All the lines arranged from top to bottom in the increasing order of time. The top subfigure is at density $\rho^{*}=0.295$, middle subfigure is at density $\rho^{*}=0.305$, and bottom subfigure is at density $\rho^{*}$ $=0.315$.

of the power law obtained from the slope of the fit is 0.79 . The observation of power law in the first shell of the relaxation confirms that decay of pseudonematic regions of size of first nearest neighbor distance also shows power law relaxation.

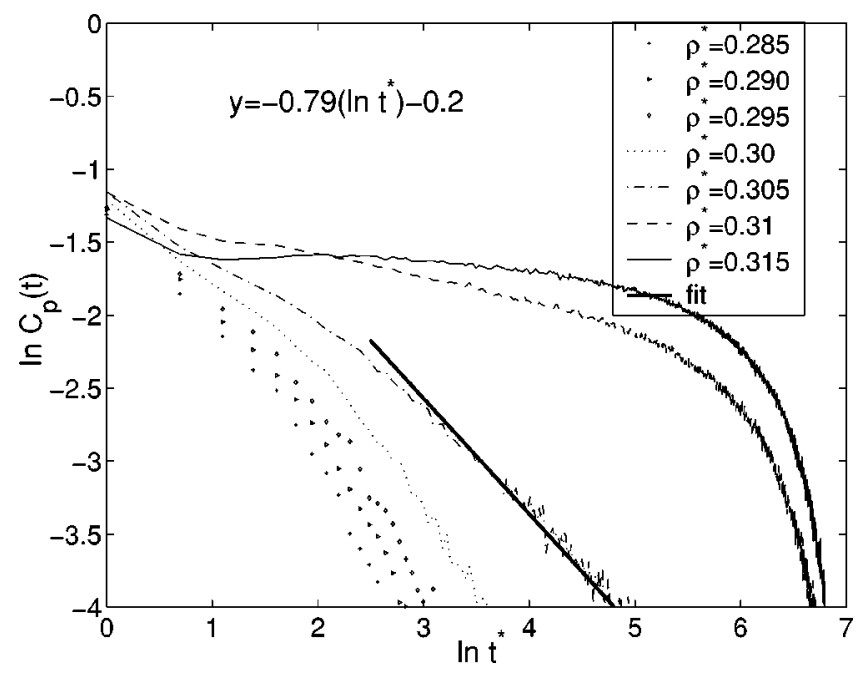

FIG. 8. The log of pair time correlation function $C_{p}(t)$ defined by Eq. (15) is plotted against logarithm of time at separation $R$ equal to the first peak of DOPCF. The emergence of power is evident at $\rho^{*}=0.305$. In this figure linear portion of $\ln C_{p}(t)$ vs $\ln t$ curve at density $\rho^{*}=0.305$ is fitted to a straight line. 


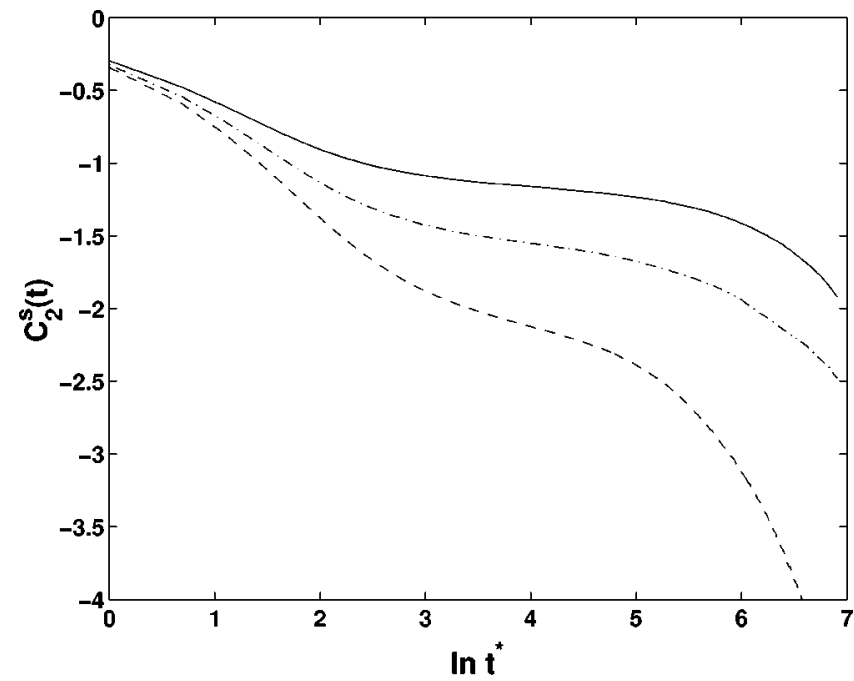

FIG. 9. The $\log -\log$ plot of the second rank single particle OTCF $\left[C_{2}^{s}(t)\right.$ in Eq. (9)] at different temperatures and at constant density $\rho^{*}=0.315$ are shown here. The continuous line gives $C_{2}^{s}(t)$ at $T^{*}=1.0$, dashed-dotted line gives $C_{2}^{s}(t)$ at $T^{*}=1.1$ and the dashed line gives $C_{2}^{s}(t)$ at $T^{*}=1.2$. The slope of the linear region varies with temperature showing temperature dependence of power law.

\section{TEMPERATURE DEPENDENCE OF RELAXATION}

In Fig. 9, the log-log plot of single particle OTCF is shown at three different temperatures $\left(T^{*}=1.0,1.1\right.$, and 1.2). At all temperatures this OTCF slows down significantly and takes long time to relax. The emergence of the power law is indicated by the linear region of the second rank OTCF. Note that when temperature increases the slope of the linear region of the second rank single particle OTCF decreases, showing the temperature dependence of power law exponent. In Fig. 10, the log-log plot of collective OTCF is plotted at three different temperatures $\left(T^{*}=1.0,1.1\right.$, and 1.2). Note that even at the high temperature 1.2 the decay of

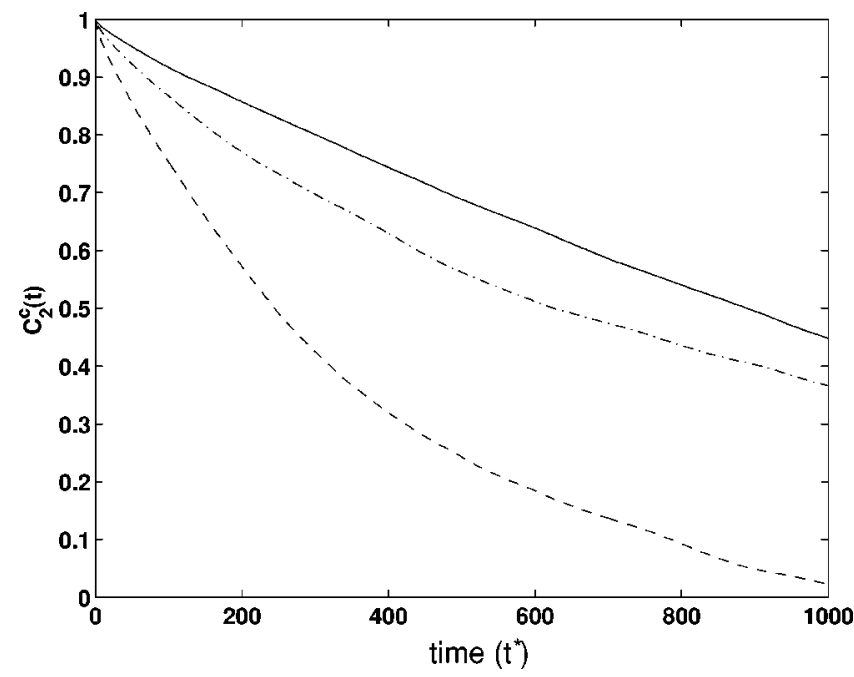

FIG. 10. This figure shows the time dependence of collective second rank OTCF $\left[C_{2}^{c}(t)\right.$, defined in Eq. (10)] at different temperatures and at constant density $\rho^{*}=0.315$. The continuous line gives $C_{2}^{c}(t)$ at $T^{*}=1.0$, dasheddotted line gives $C_{2}^{c}(t)$ at $T^{*}=1.1$ and the dashed line gives $C_{2}^{c}(t)$ at $T^{*}$ $=1.2$.

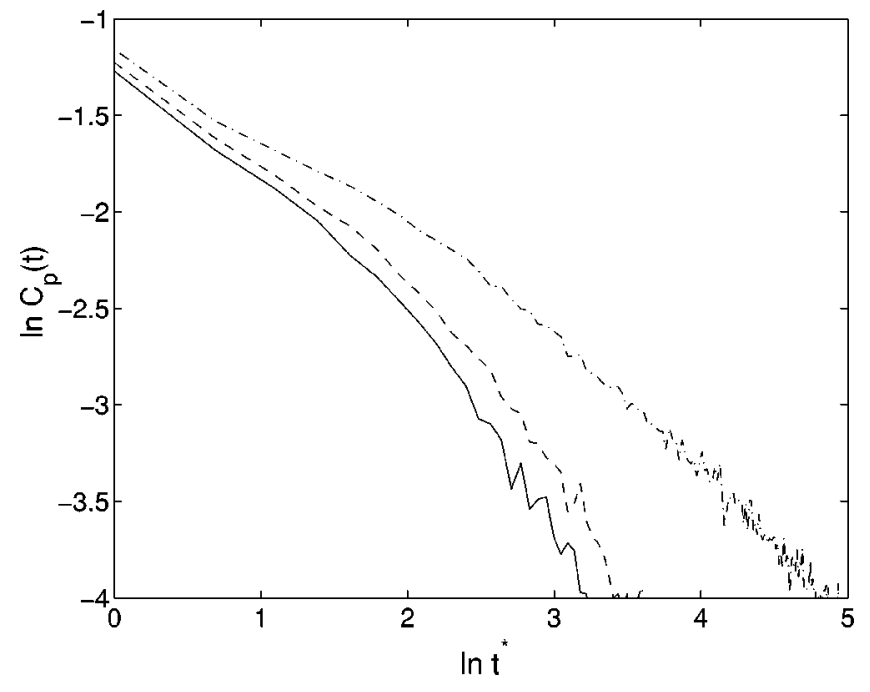

FIG. 11. The $\log$ of the pair time correlation function $C_{p}(t)$ [Eq. (15)] is plotted against $\log$ of time at three different temperature at density $\rho^{*}$ $=0.305$. The dashed-dotted line gives $C_{p}(t)$ at $T^{*}=1.0$, the dashed line gives $C_{p}(t)$ at density $T^{*}=1.1$ and the $C_{p}(t)$ at $T^{*}=1.2$ is given by continuous line.

this correlation function is slow. This also points toward the fact that even the long time simulation used here is inadequate to probe the full decay of this correlation function. It is also evident from the phase diagram (Fig. 1) that transition point has only a small variation due to change of temperature. The $\log$ of the pair time correlation function $C_{p}(t)$ in a system of density $\rho^{*}=0.305$ is plotted against log of time at three different temperatures in Fig. 11.

\section{CONCLUDING REMARKS}

In this paper extensive and long molecular dynamics simulations of orientational relaxation of model nematogens have been carried out in a system of Gay-Berne ellipsoids in an attempt to understand the origin of the experimentally observed temporal power law decay of the second rank orientational order parameter in the isotropic phase of a nematic liquid crystal. We have observed the well-known divergence of the angular pair correlation functions as the system goes toward INPT along the density axis. This divergence gives evidence of the formation of nematic domains inside the isotropic phase. The single particle second rank orientational time correlation function (OTCF) shows the emergence of a power law in the relaxation beyond the density 0.3 . The power law exponent obtained from the linear fit of the second rank single particle OTCF is $\sim 0.7$.

Perhaps the most important result of this work is the finding that the second rank collective OTCF shows an abrupt emergence of a power law decay very close to the isotropic-nematic phase transition. In fact, over a substantial part of its short time decay, the correlation function decays almost linearly with time, predicting a very weak time dependence of the derivative of $C_{2}^{c}(t)$-it is the time derivative is that measured in experiments. In the very long time, the decay becomes exponential-like, in agreement with the Landau-de Gennes mean-field theory (which describes relaxation in terms of randomization of pseudonematic do- 
mains). It is certainly heartening to observe the appearance of the power law in the decay of the collective correlation function. Note that all the earlier studies have failed to observe this power law decay because these early simulations were all limited to rather short times. In fact, the power law was found in the single particle correlation function because the latter decays on a much shorter time scale.

Mode coupling theory analysis of this problem (presented earlier) suggests that not only the orientational pair correlation function must grow rapidly (with temperature or density) as the INPT is approached, but also the long wave number part of the Fourier transformed static pair correlation function must become very large to make a dominant contribution to the memory function. ${ }^{28,31}$ This happens at very small wave number. Thus, the success in capturing in simulations of many aspects of the interesting dynamical behavior near the isotropic-nematic transition should be attributed to the carrying out of very long simulations.

Another notable new result is the observed slow relaxation in the orientational pair correlation function. Over a limited range of time, this relaxation function can be fitted to a power law decay when the separation between the pairs is equal to the first maximum in the static pair correlation function. The existence of power law at the level of pair correlation function shows that even two particle relaxation slows down at nearest neighbor level. Further work is required to fully understand the nature of these time correlation functions.

As noted earlier, there are certain striking similarities between relaxation near INPT (in the isotropic phase) of a liquid crystalline system and that in supercooled liquids. ${ }^{17,19}$ It has been found that the same functional form can describe relaxation in both supercooled liquids and in isotropic liquid of liquid crystals. The nature of the relaxation function is similar in both the systems - a short time decay, followed by a pronounced power law which is again followed by a slow exponential-like decay. As the temperature is lowered toward the glass transition temperature (for supercooled liquids) or toward the INPT, the duration of the power law increases. ${ }^{19}$ While the dynamics appear to be quite similar, there are also some differences, especially in the origin of the behavior. In the case of liquid crystals, the behavior is certainly due to the rapid growth in the equilibrium orientational pair correlation function which is reflected in the rapid increase in the long wave number limit of the orientational structure factor. This is reflected in the large growth of $S_{220}(k=0)$ near the INPT. This growth also gives rise to slow down of the mean-field Landau-de Gennes relaxation time, $\tau_{\mathrm{LdG}}$, as given by Eq. (2) and discussed earlier. In the case of supercooled liquid, however, the slow down of relaxation at intermediate wave numbers, when the wave number $k \approx 2 \pi / \sigma$, where $\sigma$ is the molecular diameter. The mean-field theory again gives a decay at intermediate wave numbers given by $S(k) / D$ where $S(k)$ is the static structure factor at intermediate wave numbers while $D$ is the self-diffusion coefficient. The large growth in relaxation time in supercooled liquid is due to a feedback mechanism ${ }^{36-39}$ where slow down of density relaxation increases friction and the increase of friction causes further slowdown of the density relaxation. This feedback mechanism is included through a self-consistent solution which gives rise to the well-known power laws and the eventual divergence of the relaxation time in the mode coupling theory. It should also be noted here that the mode coupling theory breaks down completely at low temperatures because it predicts a critical point (and divergence of viscosity and relaxation time) at a temperature which is about $30-50 \mathrm{~K}$ above the true glass transition temperature. However, prior to this breakdown, MCT is known to provide a satisfactory description of the relaxation behavior. Therefore, it is clear that while the power law relaxation near INPT has a thermodynamic origin, the ones in the supercooled liquid has a dynamic origin (note that MCT predicts a dynamic transition only).

Nevertheless, experiments have unequivocally shown that the two relaxation are quite similar. The reason may be that the basic features simply cannot be too different. Both must have an initial fast decay (due to short range interactions), followed by a slow power-law decay (due to different reasons though) and both have a long time exponential-like decay. Thus, the basic features of relaxations are the same and, therefore, can be described by the same functional form. Even with these observations, experimental observations of the similarity is indeed revealing.

We have already discussed the mode coupling theory interpretation of the slow decay. It seems that the basic conclusions of MCT is in agreement with the simulation results. It will be interesting to use the two particle correlation functions directly from simulations and perform a more detailed comparison between theory and simulation results. In addition, we need a fully self-consistent MCT, which is not attempted yet. Work in this direction is under progress.

Also, even with our very long MD simulations, it is hard to study the crossover regions with definitive conclusions. For that to occur, we need to simulate much larger systems (may be something of the order of 5000 particles) for similarly long times. Also, we have not addressed the aspect ratio dependence of the power law behavior. We hope to address these issues in future.

\section{ACKNOWLEDGMENTS}

It is a pleasure to thank Professor Michael Fayer (Stanford University) for discussions and correspondence on many aspects of power law decay. The authors also thank R. Vasanthi and Dr. Steve Gottke for collaboration. This work was supported in parts by grants from Department of Science and Technology and Council of Scientific and Industrial Research, India.

\section{APPENDIX: ORIENTATIONAL RELAXATION OF A LOCAL DIRECTOR}

The definition of yet another OTCF can be done in terms of the local director the ellipsoids. This definition is important in the case of experiments where a small fraction of molecules are excited and the observation of the collective orientational relaxation of this fraction of molecules by probe pulse. Let there be a unit vector associated with each 


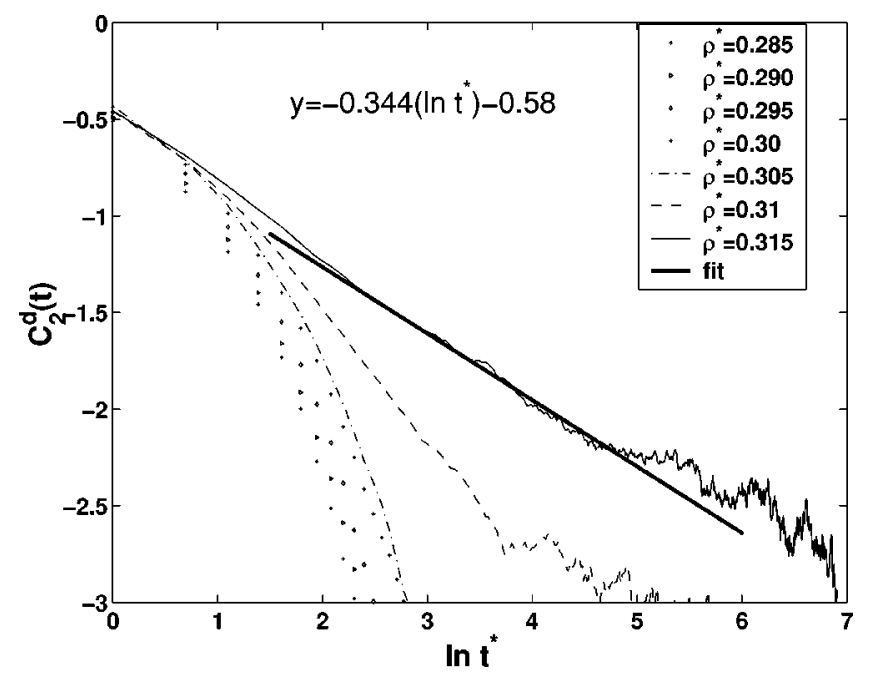

FIG. 12. The $\log -\log$ plot of second rank OTCF of the local director, $\left[C_{2}^{d}(t)\right.$, Eq. (A2) $]$ vs time at different densities and at constant temperature $T^{*}=1$ is shown in this figure. The linear portion of the curve found after $\rho^{*}=0.3$ shows the emergence of power law. The linear fit of the second rank OTCF of the local director $\left[C_{2}^{d}(t)\right]$ at $\rho^{*}=0.315$ is shown in the figure.

ellipsoid along the major axis. In a system of ellipsoids it is possible to define a local director in terms of the sum of the unit vectors. Since there is no asymmetry along the axis in the interacting potential sum of unit vectors that initially has a direction will vanish due to the rotation of the directors. This rotational diffusion of the ellipsoid can be modeled in terms of a symmetric double well potential. The arbitrary initial direction of the ellipsoid is in the first well of this symmetric double-well potential and a rotation of $\pi$ from the initial direction, which is indistinguishable from this initial direction is the other well of the symmetric double-well potential. For the $\pi$ rotation the ellipsoid has to over come the potential barrier that is created by it is neighbors. Correlation time can be defined in terms of the relaxation time of the initial direction of the resultant vector. This can also be defined in terms of principle of conservation of angular velocity in the system. The resultant orientation of the system is given by

$$
\hat{U}=\frac{\sum_{i=1}^{N} \hat{e}_{i}}{\left|\sum_{i=1}^{N} \hat{e}_{i}\right|} .
$$

Hence the $l$ th rank OTCF is defined as

$$
C_{l}^{d}(t)=\frac{\left\langle P_{l}(\hat{U}(t) \cdot \hat{U}(0))\right\rangle}{\left\langle P_{l}(\hat{U}(0) \cdot \hat{U}(0))\right\rangle} .
$$

The angular velocity $\vec{\omega}$ is conserved locally in a system at equilibrium, so $\sum_{i=1}^{N} \vec{\omega}_{i}=$ constant. In a simulation to avoid the rotational flows in the box this constant is equal to zero. In the isotropic region all the ellipsoids are free to rotate individually, hence the direction of the resultant unit vector is changing fast. But near INPT the rotational motion of the individual ellipsoids are restricted by the formation of pseudonematic regions. Hence the orientational relaxation of the

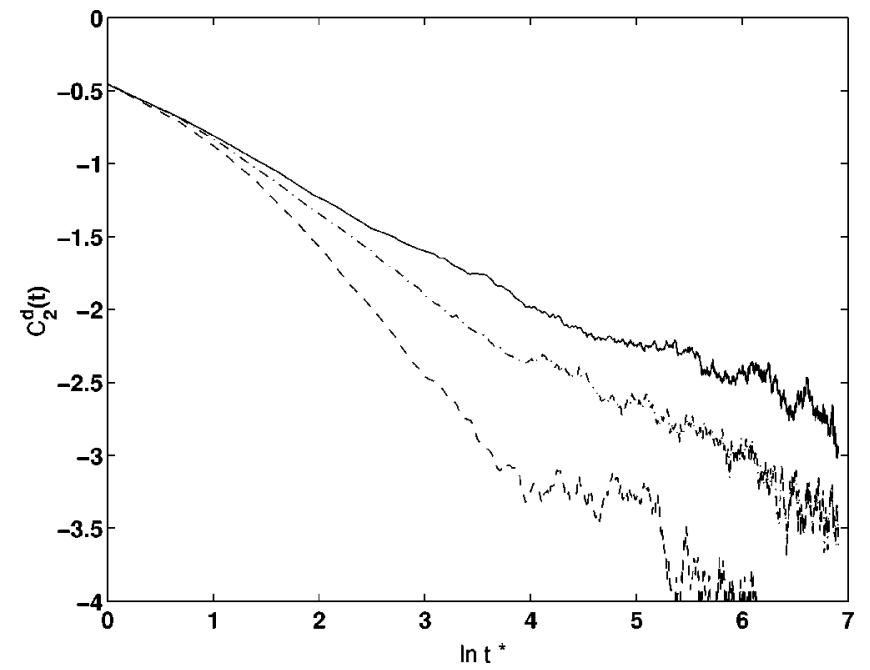

FIG. 13. The time dependence of second rank of the OTCF of the local director $\left[C_{2}^{d}(t)\right.$, defined in Eq. (A2)], is plotted at different temperatures and constant density $\rho^{*}=0.315$. The continuous line gives $C_{2}^{d}(t)$ at $T^{*}=1.0$, dashed-dotted line gives $C_{2}^{d}(t)$ at $T^{*}=1.1$ and the dashed line gives $C_{2}^{d}(t)$ at $T^{*}=1.2$.

resultant unit vector $\hat{U}$ slows down. Note that in the nematic region the molecules are aligned in one direction due to the restricted individual rotations. Therefore, the relaxation of each arbitrarily assigned unit vector associated with the ellipsoid also slows down, which results in the delay in the relaxation time the local director $\hat{U}$. The long tail of the $C_{l}^{d}(t)$ due to the forbidden rotation by formation of the nematic alignment which follows after the initial decay, results in power law behavior. We believe that the power law relaxation of this second rank orientational time correlation function of the local director may be related to the experimental results, ${ }^{16,17}$ as follows. Here for measuring the relaxation of local director, the ellipsoids are given an arbitrary initial direction. The relaxation time during which this average director loses the memory of the initial direction depends on the relaxation time of individual ellipsoids. It can be considered as loss of polarizability of a small region of the system of molecules due to random rotation.

The $\log -\log$ plot of second rank OTCF of the local director of a system of ellipsoids at different densities $\rho^{*}$ $=0.285$ to 0.315 at $T^{*}=1$ near INPT is shown in Fig. 12 . This figure shows that the relaxation of the second rank OTCF of the local director exhibits a pronounced power law decay over a rather long time interval. At higher densities (beyond $\rho^{*}=0.305$ ) a long tail appears in the second rank OTCF of the directors due to enhancement of collective interactions. After this particular density the change in the correlation function becomes dramatic and the relaxation becomes extremely slow. In Fig. 12 the linear potion of the second rank orientational time correlation function of the directors is fitted to a straight line at density 0.315 . The power law exponent obtained from this fit is 0.34. In Fig. 13 the $\log -\log$ plot of OTCF of the local director is shown at three different temperatures $\left(T^{*}=1.0,1.1\right.$, and 1.2). This correlation function shows a long linear region showing the emergence of power laws. 
${ }^{1}$ L. Onsager, Ann. N.Y. Acad. Sci. 51, 621 (1949).

${ }^{2}$ R. Zwanzig, J. Chem. Phys. 39, 1714 (1963).

${ }^{3}$ P. G. de Gennes, Phys. Lett. A 30, 454 (1969).

${ }^{4}$ P. G. de Gennes, The Physics of Liquid Crystals (Clarendon, Oxford, 1974).

${ }^{5}$ S. Chandrasekhar, Liquid Crystals (Cambridge University Press, Cambridge, 1977).

${ }^{6}$ M. J. Stephen and J. P. Straley, Rev. Mod. Phys. 46, 617 (1974).

${ }^{7}$ H. Mulder and D. Frenkel, Mol. Phys. 55, 1193 (1985).

${ }^{8}$ A. Perera, G. N. Patey, and J. J. Weis, J. Chem. Phys. 89, 6941 (1988).

${ }^{9}$ Y. Singh, Phys. Rev. A 30, 583 (1984).

${ }^{10}$ J. P. Singh and Y. P. Singh, Phys. Rev. A 33, 2725 (1986).

${ }^{11}$ E. DeMiguel, L. F. Rull, M. K. Chalam, K. E. Gubbins, and F. Van Swol, Mol. Phys. 72, 593 (1991).

${ }^{12}$ M. Baus, J. L. Colot, X. G. Wu, and H. Xu, Phys. Rev. Lett. 59, 2184 (1987).

${ }^{13}$ J. L. Colot, X. G. Wu, H. Xu, and M. Baus, Phys. Rev. A 38, 2022 (1988).

${ }^{14}$ J. J. Stankus, R. Torre, and M. D. Fayer, J. Phys. Chem. 97, 9478 (1993).

${ }^{15}$ A. Sengupta and M. D. Fayer, J. Chem. Phys. 102, 4193 (1995).

${ }^{16}$ S. D. Gottke, D. D. Brace, H. Cang, B. Bagchi, and M. D. Fayer, J. Chem. Phys. 116, 360 (2002).

${ }^{17}$ S. D. Gottke, H. Cang, B. Bagchi, and M. D. Fayer, J. Chem. Phys. 116, 6339 (2002).

${ }^{18}$ H. Cang, J. Li, and M. D. Fayer, Chem. Phys. Lett. 366, 82 (2002).

${ }^{19}$ H. Cang, J. Li, V. N. Novikov, and M. D. Fayer, J. Chem. Phys. 118, 9303 (2003).

${ }^{20}$ P. Martinoty, S. Candau, and F. Debeauvais, Phys. Rev. Lett. 27, 1123 (1971).
${ }^{21}$ M. P. Allen and D. Frenkel, Phys. Rev. Lett. 58, 1748 (1987).

${ }^{22}$ A. Perera, S. Ravichandran, M. Moreau, and B. Bagchi, J. Chem. Phys. 106, 1280 (1997).

${ }^{23}$ M. P. Allen and M. A. Warren, Phys. Rev. Lett. 78, 1291 (1997).

${ }^{24}$ S. Ravichandran, A. Perera, M. Moreau, and B. Bagchi, J. Chem. Phys. 107, 8469 (1997); 109, 7349 (1998).

${ }^{25}$ R. Vasanthi, S. Ravichandran, and B. Bagchi, J. Chem. Phys. 115, 10022 (2001).

${ }^{26}$ C. Grey and K. E. Gubbins, Theory of Molecular Fluids (Clarendon, Oxford, 1984).

${ }^{27}$ J. P. Hansen and I. R. McDonald, Theory of Simple Liquids (Academic, London, 1986).

${ }^{28}$ B. Bagchi and A. Chandra, Adv. Chem. Phys. 80, 1 (1991).

${ }^{29}$ D. J. Tildesley and P. A. Madden, Mol. Phys. 48, 129 (1983).

${ }^{30}$ B. J. Berne and R. Pecora, Dynamic Light Scattering: With Applications to Chemistry, Biology and Physics (Wiley, New York, 1976).

${ }^{31}$ J. G. Gay and B. J. Berne, J. Chem. Phys. 74, 3316 (1981).

${ }^{32}$ J. Crain and A. V. Komolkin, Adv. Chem. Phys. 109, 39 (1999).

${ }^{33}$ W. B. Streett and D. J. Tildesley, Proc. R. Soc. London, Ser. A 355, 239 (1972).

${ }^{34}$ M. P. Allen and D. J. Tildesley, Computer Simulation of Liquids (Clarendon, Oxford, 1987).

${ }^{35}$ J. M. Haile and C. G. Grey, Chem. Phys. Lett. 76, 583 (1980).

${ }^{36}$ T. Geszti, J. Phys. C 16, 5805 (1983).

${ }^{37}$ E. Leutheusser, Phys. Rev. A 29, 2765 (1984).

${ }^{38}$ T. R. Kirkpatrick, Phys. Rev. A 31, 939 (1985).

${ }^{39}$ W. Götze and L. Sjögren, Rep. Prog. Phys. 55, 241 (1992). 